\title{
Colitis complicating chronic granulomatous disease. A clinicopathological case report
}

\author{
J M Sloan, C H S Cameron, R J Maxwell, D R McCluskey, J S A Collins
}

\begin{abstract}
Background-This report concerns a female patient now aged 24 years, diagnosed at the age of 7 years as suffering from chronic granulomatous disease. At age 20 she developed diarrhoea accompanied by rectal bleeding. Endoscopy showed extensive colitis. She failed to respond to medical treatment and underwent total colectomy two years later.

Aims-To discuss the histological changes in the colon in chronic granulomatous disease.

Results-There was extensive mucosal inflammation throughout colon and rectum resembling ulcerative colitis. In addition characteristic large pigmented macrophages were distributed in the basal mucosa and superficial submucosa. Similar cells accompanied by granulomata were present in mesenteric lymph nodes. Conclusions-Colitis is an unusual clinical manifestation of chronic granulomatous disease but the presence and characteristic distribution of such pigmented macrophages in colonic biopsy in children and young adults may suggest the diagnosis. (Gut 1996; 38: 619-622)
\end{abstract}

Keywords: chronic granulomatous disease, colitis, histopathology.

Chronic granulomatous disease is an inherited condition characterised by recurrent infections resulting from an inability of phagocytic cells to kill some types of ingested bacteria and fungi. Catalase producing bacteria, principally Staphylococcus aureus are the most common cause of infection. The disease is usually $\mathrm{x}$-linked affecting male infants and presenting with widespread infections of lung, skin, lymph nodes liver, bones and joints, middle ear and nasal sinuses. The disease may also show autosomal recessive pattern of inheritance affecting female as well as male infants and may present later in childhood or even in early adulthood. These cases are often less severe. ${ }^{12}$ Gastrointestinal manifestations are not common but several reports have appeared over the past 20 years. ${ }^{3-5}$ This report concerns a further case in which colitis and diarrhoea developed 16 years after the initial diagnosis and eventually necessitated total colectomy.

\section{Case report}

Clinical history

The patient is a young woman aged 24 years.
She first presented at the age of 7 with intermittent painful swellings in the lower jaw, which continued over several years and resolved spontaneously or after penicillin treatment. At the age of 10 years, tests of immune function showed impaired ability of leucocytes to kill ingested $S$ aureus organisms and failure of neutrophils to reduce dye in the nitroblue tetrazolium test. A diagnosis of chronic granulomatous disease was made and long term treatment with co-trimoxazole (Septrin) was started.

The patient remained fairly well for 10 years but at age 20 developed some rectal bleeding. Colonoscopy showed inflammation of the rectal mucosa and at this time she had iron deficiency anaemia (haemoglobin $8.9 \mathrm{~g} / \mathrm{l}$ ). Shortly afterwards persistent diarrhoea developed with continuing chronic blood loss. Stool cultures were carried out on several occasions but no pathogens were isolated. As the patient was receiving prophylactic co-trimoxazole the possibility of post-antibiotic colitis was considered and a course of vancomycin was started. This did not result in any improvement. A year later repeat colonoscopy showed continuing rectal inflammation with sessile umbilicated lesions. Topical and systemic corticosteroid treatment was started. Shortly after this azathioprine was added to the treatment regimen. A year later, however, the patient was admitted as an emergency with severe bloody diarrhoea (12 movements per day) and weight loss. Colonoscopy at this stage showed mucosal inflammation as far as the hepatic flexure.

Abdominal colectomy and ileostomy was carried out when the patient was aged 22. After this the patient's condition improved and she gained weight. A year later she complained of anal discomfort and bloody discharge from the rectal stump. Shortly afterwards abdominoperineal excision of the rectal stump was carried out.

\section{Pathological examination}

Macroscopic examination of the colectomy specimen showed less abnormality than expected. Mucosal folds were prominent and the mucosa was thickened and oedematous. Abnormal ulceration was not seen and there were no pseudopolyps or strictures.

Histological examination showed acute and chronic mucosal inflammation throughout the colon and rectum. This varied in intensity, when severe it was accompanied by small mucosal erosions, occasional crypt abscesses, and mucosal gland distortion. Inflammation was diffuse throughout the mucosa. Plasma 


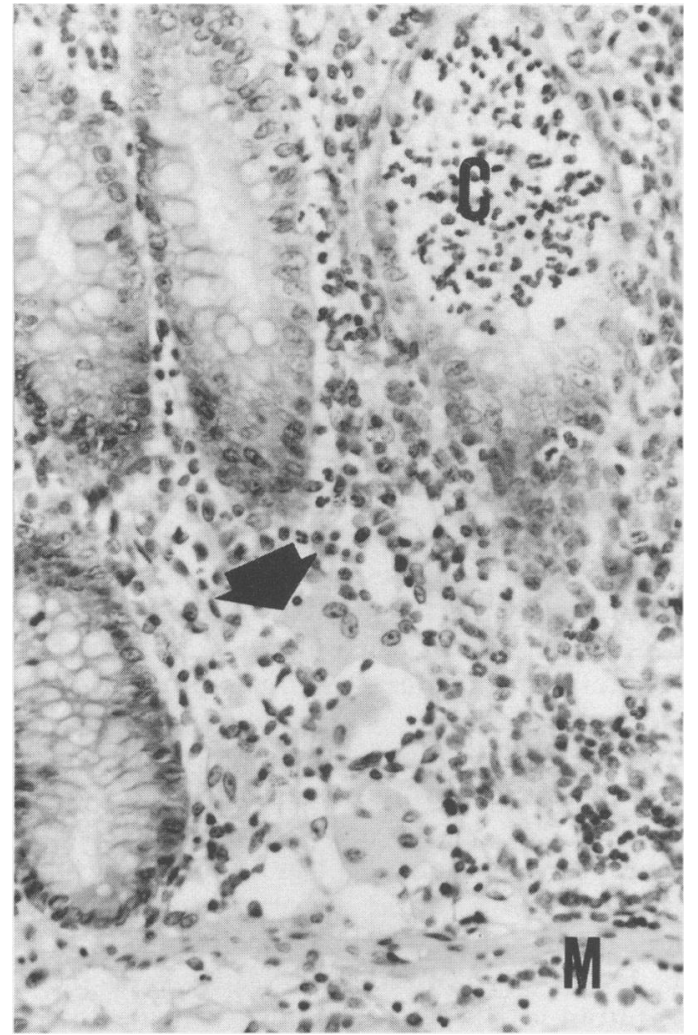

Figure 1: Pigmented macrophages (arrow) within inflamed colonic mucosa adjacent to the muscularis mucosa (M) (C) Is a crypt abscess. Original magnification $\times 125$.

cells were evenly distributed and there was not abnormal basal plasmacytosis. Inflammation was largely confined to the mucosa or superficial submucosa and was not transmural. No deep ulceration was present. In places the mucosal surface was villiform. Lymphoid aggregates were prominent, straddling the muscularis mucosa or in the superficial submucosa. Occasional giant cells were present within the lamina propria but these were adjacent to damaged mucosal glands and true granulomata were not identified within the gut wall. Lightly pigmented, large, pale brown staining macrophages were present throughout the colon and rectum. In the large intestine these were distributed almost exclusively in the lamina propria of the basal mucosa (Fig 1) and within lymphoid aggregates close to the

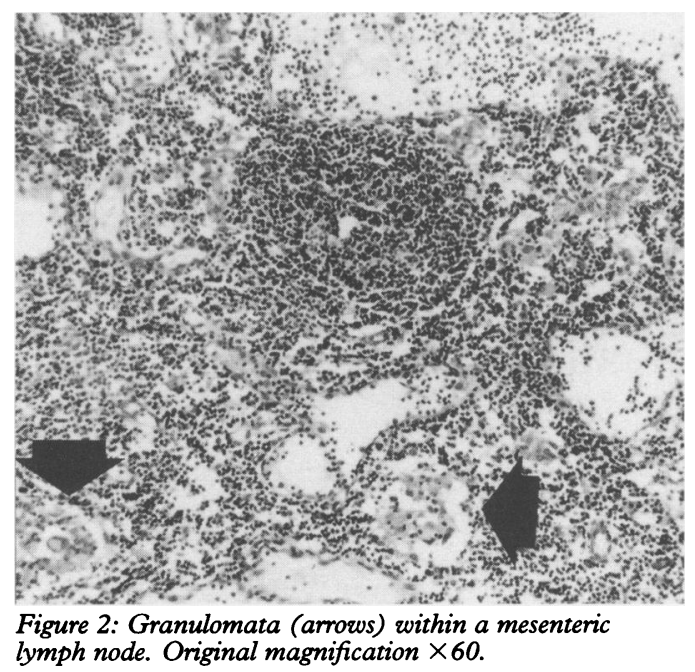

muscularis mucosa. They were present in all sections examined but were more obvious in areas showing less active inflammation. They stained faintly positive with periodic acidSchiff (PAS) and Masson Fontana stains but negative with Perl's prussian blue. Sections taken at the proximal limit of resection showed similar macrophages within the lamina propria of the terminal ileum but in contrast with the colon they were mainly found at the tips of the villi rather than in the basal mucosa.

Numerous enlarged lymph nodes were present in the mesenteric fat adjacent to the colon. In contrast with the bowel wall many of these contained numerous ill defined noncaseating granulomata with occasional giant cells (Fig 2). Small numbers of pigmented macrophages similar to those seen in the colonic mucosa were also present. ZiehlNeelsen, Grocott, and PAS stains failed to show any organisms.

Electron microscopy was carried out on a rectal biopsy specimen taken before colectomy. It showed focal mucosal erosions with invasion by normal appearing polymorphs. The macrophages in the deep mucosa described above, contained cytoplasmic lipid vacuoles and electron dense bodies identical with lipofuchsin (Fig 3). Aggregated polymorphs covered parts of the mucosal surface. These contained ingested bacteria in various stages of degradation. Some polymorphs contained apparently viable bacteria despite obvious depletion of lysosomal granules (Fig 4). No fungi, parasites or viral particles were identified.

Histological examination of the rectal stump removed a year later showed similar features but with more intense active mucosal inflammation, erosions, and glandular distortion. The most striking feature was a considerable increase in intramucosal lymphoid hyperplasia. Granulomata were again not identified. These findings were interpreted as evidence of diversion colitis superimposed on the pre-existing condition. ${ }^{6}$

\section{Discussion}

Manifestations of chronic granulomatous disease are caused by inability of the patient's phagocytic cells to kill some types of ingested micro-organisms. ${ }^{7}$ As a result the disease is characterised by recurrent infections affecting many organs but especially lungs, lymph nodes, skin and soft tissues and less often liver, gut, and bone. $S$ aureus is the organism most frequently involved and together with aspergillus accounts for the great majority of infective episodes. ${ }^{8}$ The disease is inherited as $x$-linked recessive or autosomal recessive and the associated genetic defects have been well characterised. ${ }^{10} \mathrm{x}$-Linked disease affects male infants, presents early in life, and is frequently fatal before puberty if untreated. The autosomal recessive pattern of inheritance often presents later in childhood, may affect girls, and is frequently much less severe. ${ }^{2}$

Neither of our patient's parents showed abnormality of the nitroblue tetrazolium test. This does not exclude, however, either the 


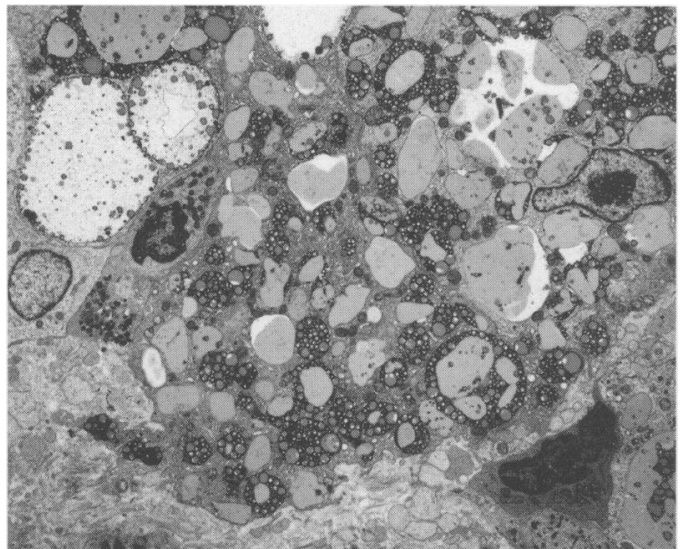

Figure 3: Electron photomicrograph. Numerous lipid droplets and lipofuscin granules fill the cytoplasm of a collection of macrophages within the lamina propria of colonic mucosa. Original magnification $\times 3400$.

$x$-linked or autosomal carrier states. ${ }^{10}$ Thus it is uncertain if this case represents an example of inherited disease or of spontaneous germline mutation, which may also occur. ${ }^{11}$

There have been several histological reports of intestinal involvement in chronic granulomatous disease ${ }^{3412}$ most of which describe granulomatous lesions within the gut wall suggestive of Crohn's disease. Werlin et $a l^{5}$ gave a detailed description of the relevant colonic histology and described granulomata in the mucosa and submucosa, abscesses and mucosal infiltration by pigmented histiocytes as the most salient features.

This case report shows many similarities but despite examination of numerous sections, granulomata were not seen within the gut wall despite their obvious presence in mesenteric lymph nodes. The histological appearances of the mucosa were more suggestive of ulcerative colitis than Crohn's disease, in view of the continuous inflammation throughout the colon and rectum, restriction of inflammation to the mucosa, absence of granulomata and, in places, villiform appearance of the mucosal surface. The distinctive features were the presence of lightly pigmented macrophages in the lamina propria adjacent to the muscularis mucosa and the increased amount of lymphoid

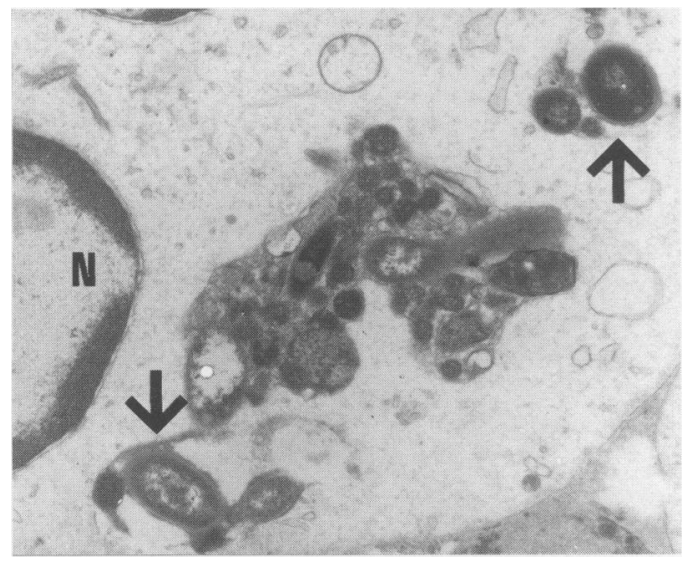

Figure 4: Electron photomicrograph. Partially degraded and intact bacteria (arrow) are contained within the cytoplasm of a polymorph, which is almost devoid of cytoplasm of a polymorph, which is almost devoid of
lysosomes and other organelles. (N) Is a lobe of the cell nucleus. Original magnification $\times 16000$. tissue also in that region. The macrophages were most prominent in areas where inflammation was less intense. They were not found in the upper mucosa but were also seen within lymphoid aggregates in the superficial submucosa. Similar cells are found in chronic granulomatous disease in various organs including lymph nodes, liver, and lung. Electron microscopy of these cells showed features similar to those described by Park and Chi. ${ }^{13}$ Lipofuchsin granules were present throughout the cytoplasm together with lipid droplets. As an additional finding we demonstrated fragments of ingested bacteria within the cytoplasm of degranulated polymorphs. Some of the bacteria appeared viable and would be consistent with the failure of phagocytic cells to kill certain ingested bacteria, which is characteristic of the disease.

Fisher et al ${ }^{14}$ described a case of ulcerative colitis complicating chronic granulomatous disease in a 10 year old boy. Their description of the colonic pathology is similar to our case but lesions were more severe with extensive mucosal ulceration. It is difficult to be sure if these cases represent two concomitant disease processes or are simply a manifestation of chronic granulomatous disease affecting the colon. There is now considerable evidence that bacterial cell wall products act as stimulatory factors in inflammatory bowel disease. This is accompanied for reasons as yet unknown by subsequent upregulation of proinflammatory cytokines. ${ }^{15}$ The persistence of viable bacteria within phagocytic cells in colonic mucosa in chronic granulomatous disease may have a similar effect causing excessive stimulation of the inflammatory process with subsequent mucosal damage.

Scattered pigmented and non-pigmented PAS positive macrophages are frequently seen within the lamina propria of colonic mucosa. These are often not associated with obvious disease and are probably of no clinical significance. These cells are often located in the superficial mucosa in contrast with the larger macrophages seen in chronic granulomatous disease, which are predominantly found in the basal mucosa.

Large numbers of pigmented macrophages within the lamina propria of the colon are usually a manifestation of melanosis coli. ${ }^{16}$ The macrophages in chronic granulomatous disease are localised to the basal mucosa adjacent to the muscularis mucosa and within lymphoid aggregates while those of melanosis coli tend to occur in the upper mucosa. The pigment in chronic granulomatous disease is considerably lighter than in melanosis coli and would easily be missed on haematoxylin and eosin staining. The dark pigmentation of melanosis coli associated with anthranoid laxative abuse usually occurs in middle aged and elderly patients. Other laxatives, however, may induce lighter pigmentation. ${ }^{17}$ Iron containing pigmented macrophages associated with previous mucosal ulceration or haemorrhage may be identified by a positive Perl's stain. Barium containing macrophages may also be found within colonic mucosa but these usually have 
a pale green tinge and are doubly refractile. Whipple's disease affecting the gastrointestinal tract may be associated with the presence of foamy macrophages in the lamina propria of colonic mucosa. These are strongly PAS positive and are usually found in the superficial mucosa. ${ }^{16}$ In the small intestine after treatment, localisation of these cells may change and they may be found close to the muscularis mucosa. ${ }^{18}$ It is uncertain if a similar change in localisation occurs in the colon. However, Whipple's disease affects a much older age group than chronic granulomatous disease and electron microscopy shows the macrophages to contain large numbers of bacilli.

In conclusion we report a case of colitis associated with chronic granulomatous disease in a young women necessitating colectomy. The finding of light brown pigmented macrophages in colonic mucosa adjacent to the muscularis mucosa with or without granulomata, in a young patient should raise the possibility of a diagnosis of chronic granulomatous disease affecting the bowel especially if there is a history of recurrent infection.

1 Tauber AI, Borregaard N, Simons E, Wright J. Chronic granulomatous disease: a syndrome of phagocyte oxidase granulomatous disease: a syndrome of phagc
deficiencies. Medicine 1983; 62: 286-309.

2 deficiencies. Medicine 1983; 62: 286-309. Pediatr Pathol 1990; 10: 143-53.

3 Ament ME, Ochs HD. Gastrointestinal manifestations of chronic granulomatous disease. $N$ Engl f Med 1973; 288: 382-7.

4 Yogman MW, Touloukian RJ, Gallagher R. Intestinal granulomatosis in chronic granulomatous disease and in Crohn's disease. N Engl f Med 1974; 289: 228.

5 Werlin SL, Chusid MJ, Caya J, Oechler HW. Colitis in chronic granulomatous disease. Gastroenterology 1982; 82: 328-31.

6 Warren BF, Shepherd NA. Diversion proctocolitis. Histopathology 1992; 21: 91-3.

7 Quie PG, White JG, Holmes B, Good RA. In vitro bacterocidal capacity of human polymorphonuclear leucocytes: diminished activity in chronic granulomatous disease of diminished activity in chronic granulomato

8 Gallin JI. Recent advances in chronic granulomatous disease. Ann Intern Med 1983; 99: 657-74.
disecent advances in chronic

9 Dinauer MC, Orkin SH. Chronic granulomatous disease. Annu Rev Med 1992; 43: 117-24.

10 Hopkins PJ, Bemiller LS, Curnutte JT. Chronic granulomatous disease: diagnosis and classification at the molecular level. Clin Lab Med 1992; 12: 277-304.

11 Babior BM, Woodman RC. Chronic granulomatous disease. Semin Hematol 1990; 27: 247-59.

12 Harris BN, Boles ET. Intestinal lesions of chronic granulomatous disease in childhood. 7 Pediatr Surg 1973; 8: 955-6.

13 Park SH, Chi JG. Chronic granulomatous disease: an ultrastructural study of the pigment laden histiocytes. Pediatr Ptructural study of the pigm

14 Fisher JE, Kahn AR, Heitlinger L, Allen JE, Afshani E Chronic granulomatous disease of childhood with acute ulcerative colitis: a unique association. Pediatr Pathol 1987; 7: 91-6.

15 MacDermott RP. Inflammatory bowel disease. Current Opinion in Gastroenterology 1994; 10: 355-7.

16 Talbot IC, Price AB. Biopsy pathology in colorectal disease. London: Chapman Hall, 1984: 54-7.

17 Spiessens C, de Witte P, Geboes K, Lemli J. Experimental induction of pseudomelanosis coli by anthranoid laxatives and non anthranoid laxatives. Pharmaceutical and Pharmacological Letters 1991; 1: 3-6.

18 Ectors N, Geboes K, DeVos R, Heidbuchel H, Rutgeerts P, Desmet V, et al. Whipple's disease: a histological, immunocytochemical and electron microscopy study of the immune response in the small intestinal mucosa. Histopathology 1992; 21: 1-12. 